Annals of International Medical and Dental Research

E-ISSN: 2395-2822 | P-ISSN: 2395-2814

Vol-8, Issue-2 | March-April 2022

DOI:10.53339/aimdr.2022.8.2.3

Page no- 11-14 | Section- Research Article (Ananesthesia)

\title{
Anesthetic Management of A Patient with Hypertrophic Cardiomyopathy Posted For Non-Cardiac Surgery - Case Report
}

\author{
Vidyalakshmi S1, Smriti Anand2*, B S Sekhon³, Rakesh Sadhu4
}

1Postgraduate Student, Department of Ananesthesia, Maharishi Markandeshwar Medical College and Hospital, Kumarhatti, Solan, Himachal Pradesh, India. Email ID: vidyabanu500@gmail.com

Orcid ID: 0000-0002-7085-0823

2Professor, Department of Ananesthesia, Maharishi Markandeshwar Medical College and Hospital, Kumarhatti, Solan, Himachal Pradesh, India.

Email ID: gupta.smriti1@gmail.com

Orcid ID: 0000-0001-5788-6285

3Professor, Department of Ananesthesia, Maharishi Markandeshwar Medical College and Hospital, Kumarhatti, Solan, Himachal Pradesh, India.

Email ID: drbsekhon@gmail.com

Orcid ID: 0000-0001-8297-2169

4Professor and Head, Department of Ananesthesia, Maharishi Markandeshwar Medical College and Hospital, Kumarhatti, Solan, Himachal Pradesh, India. Email ID: rsadhu2008@gmail.com

Orcid ID: 0000-0002-0287-2203

${ }^{*}$ Corresponding author

\begin{abstract}
Hypertrophic cardiomyopathy (HOCM) is a complex cardiac disorder of genetic origin. Though the patients may be asymptomatic the stress of surgery and anaesthesia is known to exacerbate the left ventricular outflow tract (LVOT) obstruction leading to catastrophic complications. We hereby report a successful anaesthetic management of a patient with left intertrochantric fracture diagnosed with HOCM. Careful and meticulous strategies to prevent LVOT obstruction led to entire uneventful introperative and perioperative course.
\end{abstract}

Received: 30 October 2021

Revised: 10 December 2021

Accepted: 23 December 2021

Published: 18 February 2022

Keywords:-Hypertrophic cardiomyopathy, Left ventricular outflow tract, Intertrochantric fracture, Spinal anaesthesia

\section{INTRODUCTION}

Hypertrophic cardiomyopathy (HCM) is a genetic disorder with autosomal dominant inheritance. It is associated with asymmetrical hypertrophy of interventricular septum culminating into periodic obstruction of the left ventricular outflow tract (LVOT) and presenting with wide range of symptomstotally asymptomatic to sudden cardiac arrest in the young due to dysrhythmias. [1] Clinical diagnosis of HCM is based on the echocardiographic findings of asymmetrical hypertrophy of the left ventricle with nondilated chamber after excluding other cardiac and systemic grounds of hypertrophy.[2] A patient presenting with HCM poses a great challenge to the attending anaesthesiologist because of the antecedent risks of intraoperative left ventricular outflow tract 
Annals of International Medical and Dental Research E-ISSN: 2395-2822 | P-ISSN: 2395-2814

Vol-8, Issue-2 | March-April 2022

DOI:10.53339/aimdr.2022.8.2.3

Page no- 11-14 | Section- Research Article (Ananesthesia)

(LVOT) obstruction, diastolic dysfunction, myocardial ischemia and dysrhythmias like atrial fibrillation or flutter. [3] We hereby report a successful anaesthetic management of a case HCM with left intertrochantric fracture posted for dynamic hip screw fixation.

\section{CASE REPORT}

$60 \mathrm{yr}$ old female weighing $52 \mathrm{~kg}$ presented in emergency department with history of fall from height and was diagnosed with left intertrochantric fracture and posted for open reduction internal fixation with dynamic hip screw.

In the preanesthetic workup a detailed history was taken and examination done. There was no history of any comorbidities though she complained of mild dyspnoea on exertion and episodes of giddiness on mild to moderate exertion. She was classified under NYHA classII. On checking her vitals, her pulse rate was 96/ minute, respiratory rate was 16/ minute, saturation $\left(\mathrm{SPO}^{2}\right)$ on room air was $98 \%$ and her blood pressure was found to be high in 2 readings 5 minutes apart : being 140/90 mmHg and $150 / 90 \mathrm{mmHg}$ respectively. Hence she was classified under JNC I. Airway examination was normal. 12-lead ECG done showed T-wave inversions in chest leads V3V6. Cardiologist opinion was sought. 2 D echocardiography (ECHO) was done for further evaluation. $\mathrm{ECHO}$ findings revealed hypertrophic cardiomyopathy-sigmoidal type, concentric Left ventricular hypertrophy, grade 1 diastolic dysfunction, normal left ventricular systolic function and the LVOT gradient was found to be $30 \mathrm{mmHg}$ at rest. Hence patient was diagnosed with HCM. Patient was started on tablet Metoprolol 50mg once daily and deep vein thrombosis prophylaxis was initiated with low molecular weight heparin. Patient was accepted under ASA III and surgery was scheduled after one week under spinalanaesthesia technique with fascia iliaca compartment block. Low molecular weight heparin was stopped $12 \mathrm{hrs}$ prior to surgery and INR was determined on the day of surgery and it was found to be 1.2. The plan of anaesthesia and the anaesthetic goals were well established prior to surgery. In the present case the chief anesthetic considerations were: To decrease LVOT (Increase inpreload,afterload, Decrease in myocardial contractility) and to curtail any kind of sympathetic stimulation, hypovolemia as well as vasodilatation which can be deleterious. Strategies to establish these were: maintenance of sinus rhythm, reduction in sympathetic activity to reduce chronotropy and inotropy, maintenance of left ventricular filling together with maintenance of systemic vascular resistance.

So the patient was premedicated with tablet Alprazolam $0.5 \mathrm{mg}$ prior to surgery to allay anxiety. Prophylactic antibiotic was administered in the preoperative ward. In the operation theatre the standard monitoring techniques comprising of ECG, non invasive blood pressure monitoring (NIBP) and pulse oximetry were applied. Her baseline vitals were noted, heart rate being $80 / \mathrm{min}$, blood pressure $130 / 80 \mathrm{mmHg}^{2}$ and $\mathrm{SPO}^{2} 98 \%$ on room air. Large bore intravenous cannula was secured. Pt was preloaded with $500 \mathrm{ml}$ of Ringer Lactate and intravenous midazolam 1mg given. Oxygen was started with Hudson mask. Under all aseptic precautions $13 \mathrm{mg}$ of hyperbaric bupivacaine with $30 \mu \mathrm{g}$ of fentanyl given in the subarachnoid space. Adequate 
Annals of International Medical and Dental Research E-ISSN: 2395-2822 | P-ISSN: 2395-2814

Vol-8, Issue-2 | March-April 2022

DOI:10.53339/aimdr.2022.8.2.3

Page no- 11-14 | Section- Research Article (Ananesthesia)

block was achieved and surgery proceeded. Urinary catheterisation was done. The hemodynamic parameters were monitored throughout the procedure. Patient had episodes of hypotension ranging from 90/70 $\mathrm{mmHg}$ to100/60 mmHg. Treated with 50-100 mcg IV bolus of phenylephrine after which blood pressure remained stable while the heart rate remained between $70-80 /$ minute and there were no episodes of tachycardia. The procedure lasted for 1.5-hour, total fluid given intraoperatively was 1.5 litres and the urine output was $150 \mathrm{ml}$. Blood loss was minimal (200ml). For post operative analgesia ultrasound guideded fascia iliaca block was given with $25 \mathrm{ml}$ of $0.3 \%$ Ropivacaine with dexamethasone $8 \mathrm{mg}$. Pain score was found to be by visual analogue scale (VAS) to be 1-2 for 12 hours. Patient was also administered injection tramadol $50 \mathrm{mg}$ six hourly together with injection paracetamol 100mg intravenous 8 hourly. Postoperative vital monitoring was strictly done and there were no episodes of hypotension or tachycardia. Patient was started on LMWH after 12 hrs of surgery and tablet metoprolol 50mg was also restarted. The patient was mobilized on the post operative day second. She was discharged on the 5 th postoperative day and the low molecular weight heparin stopped. Her entire postoperative course remained uneventful.

\section{DISCUSSION}

HOCM is a complex genetic cardiac disease. It is transmitted as autosomal dominant trait and can affect any age group. Patients can present with wide range of symptoms ranging from totally asymptomatic to symptoms of dyspnea on exertion, angina and dizziness varying from light headedness, syncope and sudden death.
Its prevalence is 1 in 500 persons and characterised by Left ventricular hypertrophy in the absence of any other cardiac disease. The common form is hypertrophy is of the interventricular septum and anterolateral free wall. Challenges for anaesthesiologist's is the high incidence of intraoperative left ventricular outflow tract (LVOT) obstruction, diastolic dysfunction, myocardial ischemia and atrial flutter, fibrillation. Pathophysiology of HCM is related to the following features: Myocardial hypertrophy, dynamic left ventricular outflow obstruction, systolic anterior movement of mitral valve causing mitral regurgitation, diastolic dysfunction, Myocardial infarction dysrhythmias.

In the present case after the diagnosis of HCM corroborated by the ECHO findings, our patient was started on beta blocker and the surgery was rescheduled after a week. Beta blockers by decreasing the heart rate and reducing the myocardial oxygen demand, prolong the diastolic filling time and are quite beneficial in HCM. Patient was also started on anticoagulants as a part of DVT prophylaxis as also a preventive measure against dysrhythmias like atrial flutter and atrial fibrillation which further enhance the risk of thromboembolism.

Though our patient had no well defined symptoms of LVOT, but stress of surgery and anaesthesia are known to provocate LVOT.[3] Though spinal anaesthesia by decreasing the afterload can exacerbate LVOT, yet the subarachnoid block was planned in the present case by adequately preloading the patient prior to administration of block and using very small doses of heavy bupivacaine and adding the adjuvant fentanyl. Also any episode of 
Annals of International Medical and Dental Research

E-ISSN: 2395-2822 | P-ISSN: 2395-2814

Vol-8, Issue-2 | March-April 2022

DOI:10.53339/aimdr.2022.8.2.3

Page no- 11-14 | Section- Research Article (Ananesthesia)

intraoperative hypotension was managed with boluses of phenylephrine which increases the systemic vascular resistance without increasing the heart rate. Patient was also administered multimodal analgesia comprising of compartment fascia iliaca block along with the systemic tramadol and paracetamol to keep the entire postoperative period pain free.

\section{CONCLUSIONS}

Hypertrophic cardiomyopathy is one of the most common causes of sudden cardiac arrest

\section{REFERENCES}

1. Patil S, Ninan B. Anaesthetic management of a patient with hypertrophic obstructive cardiomyopathy with automated implantable cardioverter defibrillator for septal myectomy with mitral valve replacement and coronary artery bypass graft. Ann Card Anaesth. 2005;8(1):55-7.

2. Maron BJ, McKenna WJ, Danielson GK, Kappenberger LJ, Kuhn HJ, Seidman CE, et al; Task Force on Clinical Expert Consensus Documents. American College of Cardiology; Committee for Practice Guidelines. European Society of Cardiology. American College of Cardiology/European Society of Cardiology clinical expert consensus document on in the young. The patients may remain asymptomatic or may present with cardiac arrythmias. The hallmark of HCM is LVOT which may manifest in an otherwise asymptomatic individual due to the stress of surgery and anaesthesia. Having a thorough knowledge of the pathophysiology of HCM can help the anaesthesiologist to sail smoothly through any possible catastrophe.

hypertrophic cardiomyopathy. A report of the American College of Cardiology Foundation Task Force on Clinical Expert Consensus Documents and the European Society of Cardiology Committee for Practice Guidelines. J Am Coll Cardiol. 2003;42(9):1687-713. doi: 10.1016/s07351097(03)00941-0.

3. PoliacLC, Barron ME, Maron BJ. Hypertrophic cardiomyopathy. Anesthesiology. 2006;104(1):183-92. doi: 10.1097/00000542-200601000-00025.

Source of Support: Nil, Conflict of Interest: None declared 